\title{
Influence of RGS2 on Sertraline Treatment for Social Anxiety Disorder
}

\author{
Murray B Stein*,', Aparna Keshaviah², Stephen A Haddad², Michael Van Ameringen ${ }^{3}$, Naomi M Simon $^{2}$, \\ Mark H Pollack ${ }^{4}$ and Jordan W Smoller ${ }^{2}$
}

'Department of Psychiatry and Family and Preventive Medicine, University of California, San Diego, La Jolla, CA, USA; '2Department of Psychiatry, Massachusetts General Hospital, Boston, MA, USA; ${ }^{3}$ Department of Psychiatry and Behavioral Neurosciences, McMaster University, Hamilton, ON, Canada; ${ }^{4}$ Department of Psychiatry, Rush University Medical Center, Chicago, IL, USA

\begin{abstract}
Only a minority of patients with social anxiety disorder (SAD) has a robust therapeutic response to evidence-based serotonin reuptake inhibitor (SSRI) treatment. To help improve the personalized medicine approach to psychiatric care, we evaluated several candidate genetic predictors of SSRI response in SAD. At the start of a randomized controlled trial (NCT00282828), 346 patients with SAD at three sites received protocol-driven, open-label treatment with sertraline, up to 200. $\mathrm{mg} / \mathrm{d}$ over 10 weeks. Efficacy was determined using a continuous measure of outcome (Liebowitz Social Anxiety Scale (LSAS)) and dichotomous indicators of response (LSAS $\leqslant 50)$ and remission (LSAS $\leqslant 30$ ). Predictors of efficacy were examined in multivariate regression models that included eight polymorphic variants in four candidate genes (four in RGS2, two in HTR2A, one in SLC6A2, and one in SLC6A4). Adjusting for genetic ancestral cluster and nongenetic predictors of response, all four single-nucleotide polymorphisms (SNPs) in RGS2 predicted change in LSAS over time, at studywise significance $(p=0.00833)$, with the minor allele associated with less improvement over time. After adjusting for genetic ancestral cluster and non-genetic predictors of remission, two of the four RGS2 SNPs predicted likelihood of remission at or just below study-wise significance $(p=0.025)$ : $r$ 4606 ( $A O R=0.49$ (95\% $C l=0.27-0.90), p=0.022)$ and $r s|8| 974 \mid(A O R=0.50(95 \% C l=0.28-0.92)$, $p=0.027$ ). Variation in RGS2, a gene previously shown to be associated with social anxiety phenotypes and serotonergic neurotransmission, may be a biomarker of the likelihood of substantially benefiting from sertraline among patients with SAD. Neuropsychopharmacology (2014) 39, 1340-1346; doi:I0.1038/npp.2013.30I; published online I2 March 2014
\end{abstract}

Keywords: social anxiety disorder; selective serotonin reuptake inhibitor; genetics; pharmacotherapy

\section{INTRODUCTION}

Social anxiety disorder (SAD; also referred to as social phobia) is a common psychiatric condition with a lifetime prevalence of $12 \%$ (Ruscio et al, 2008) and is associated with significant distress and dysfunction (Stein and Stein, 2008). Despite the availability of evidence-based treatments for SAD, many patients remain symptomatic after initial intervention. Randomized controlled trials from within the serotonin (SSRIs) and serotonin-norepinephrine reuptake inhibitors classes, which include the only FDA-approved medications for SAD, report rates of response in adults (interestingly, response rates is children are much higher (Masi et al, 2012; Wagner et al, 2004)) in the range of 40-60\%; remission rates are even lower, averaging approximately 20\% (Stein et al, 2004; Stein and Stein, 2008).

Therefore, considering the most widely used class of pharmacological treatments for SAD, SSRIs, only about half

*Correspondence: Dr MB Stein, Department of Psychiatry and Family and Preventive Medicine, Anxiety and Traumatic Stress Disorders, University of California, San Diego (Mailcode 0855), 9500 Gilman Drive, La Jolla, CA 92093-0855, USA, Tel: +858 534 6400, Fax: +858 534 6460, Email: mstein@ucsd.edu

Received 22 July 2013; revised 15 September 2013; accepted I October 2013; accepted article preview online 24 October 2013 of adult patients garner a clinically meaningful benefit (ie, 'respond') and only about half of those again derive substantial benefit (ie, 'remit') after initial intervention. It would be extremely useful if we were able to predict which patients were likely to have a substantial therapeutic response, as this would strongly influence initial choice of treatment. This is a desired aim not only for pharmacotherapy of SAD, but also for all of the mental disorders, where we lack the ability to predict the likelihood of response of a given patient to a given drug (Uher et al, 2012). We are aware of only a couple of studies that have attempted to predict response to SSRI in patients with SAD (Stein et al, 2006; Van Ameringen et al, 2004). Only one of these studies included a genetic predictor, 5HTTLPR, which in that retrospective analysis found an association between number of ' $s$ ' alleles and lower likelihood of response (Stein et al, 2006). To the best of our knowledge, no published studies have attempted to replicate that finding or to seek other genetic predictors of SSRI response in SAD.

We report here the results from analyses attempting to predict likelihood of response and remission to an SSRI coming from the first phase of a three-center trial of pharmacotherapy for SAD. This trial, Social Anxiety Pharmacotherapy Improvement, provides prospective data on a large sample of patients with SAD uniformly treated 
with the same SSRI, sertraline, on an open-label basis according to a standardized protocol.

\section{METHODS}

\section{Participants}

Participants were recruited between August 2006 and July 2011 through clinical referrals to Massachusetts General Hospital (MGH), McMaster University and University of California at San Diego, and advertisements. Inclusion criteria were: (1) male or female outpatients 18 years of age or older with a current principal diagnosis (including designation by the patient as most important source of distress or impairment) of generalized social anxiety disorder (GSAD) by Diagnostic and Statistical Manual for Mental Disorders (4th Edition) criteria, (2) a total score on the Liebowitz Social Anxiety Scale (LSAS; Fresco et al, 2001) of 60 or greater, and (3) no clinically significant medical abnormalities based on physical and laboratory examination.

Exclusion criteria included: (1) a history of more than two unsuccessful, adequate pharmacologic treatment trials, operationalized as lack of response to $\geqslant 10$ weeks of any of the following: SSRIs at adequate dose (eg, paroxetine $40 \mathrm{mg} / \mathrm{d}$ or its equivalent); benzodiazepines (eg, clonazepam $>2.5 \mathrm{mg} / \mathrm{d}$ ) plus an antidepressant (at adequate dose as above); MAOIs (eg, phenelzine $60 \mathrm{mg} / \mathrm{d}$ or its equivalent); or a single failed trial of $\geqslant 10$ weeks of venlafaxine $(\geqslant 150 \mathrm{mg} /$ d); (2) pregnant women, lactating women, and women of childbearing potential not using medically accepted forms of contraception; (3) psychotic disorders, mental retardation, organic medical disorders, bipolar disorder or OCD with YBOCS score $\geqslant 25$; recent history of eating disorders or alcohol/substance abuse or dependence. Entry of patients with major depression, panic disorder, generalized anxiety disorder, PTSD, or adult attention-deficit hyperactivity disorder (ADHD) was permitted if the GSAD was judged to be the predominant disorder; (4) concurrent use of other psychotropic medications with discontinuation of regular benzodiazepine or antidepressant therapy required at least 2 weeks ( 5 weeks for fluoxetine) before baseline: patients with comorbid adult ADHD could remain on stimulant medication if dose had been stable for $\geqslant 1$ month; (5) patients with current or recent significant suicidality; (7) any concurrent psychotherapy initiated within 3 months or any ongoing psychotherapy directed specifically toward GSAD (eg, CBT) was excluded.

\section{Study Design and Procedures}

Eligible participants were enrolled into the first phase of the study, on which the results presented here are based. Phase 1 comprised 10 weeks of open prospective treatment with sertraline initiated at $25 \mathrm{mg} / \mathrm{d}$, with all symptomatic patients titrated up to their maximally tolerated dose $(\leqslant 200 \mathrm{mg} / \mathrm{d})$ by week 8 . Participants unable to reach at least $50 \mathrm{mg} / \mathrm{d}$ by the end of Phase 1 were discontinued from the study and referred for clinical treatment. Participants were also randomly assigned to receive instructions encouraging them to interact with others while taking study medication ('exposure instructions') or instructions lacking that encouragement ('neutral instructions'); instruction set had no significant impact on Phase 1 outcome (Pollack et al, 2013). Participants were seen weekly for the first 2 weeks of Phase 1 and then bi-weekly thereafter. Efficacy was determined using a continuous measure of outcome (LSAS) and dichotomous indicators of response (LSAS $\leqslant 50$ ) and remission (LSAS $\leqslant 30$ ). At the end of week 10 , remitters were discontinued from that phase of the study and offered an additional 12 weeks of continued open-label treatment, and non-remitters were offered randomization to the second phase of the study (the results of which are described elsewhere (Pollack et al, 2013)).

A total of 397 patients received at least one dose of sertraline in the study. The present analysis is limited to the 346 patients for whom genetic and phenotypic data were available. Demographic characteristics of the subjects are shown in Table 1.

We chose to investigate a very parsimonious number of genes, and SNPs within those genes, knowing that multiple testing would limit our ability to detect study-wise significance. Candidates (Table 2) were chosen on the basis of published associations with the diagnostic phenotype (SAD) itself (Gelernter et al, 2004), with strongly related phenotypes (eg, behavioral inhibition or shyness; Arbelle et al, 2003; Smoller et al, 2008) or with SSRI response in a prior study of SAD (Stein et al, 2006).

\section{Genotyping Methods}

SNP genotyping. SNP genotyping was performed at the MGH Center for Human Genetic Research (CHGR) using the Sequenom iPLEX Gold chemistry and the MassARRAY system. The major steps in this process included the following: multiplex assay design using Assay Designer software, DNA amplification by PCR, post-PCR nucleotide deactivation using shrimp alkaline phosphatase to remove phosphate groups from unincorporated dNTPs, single-base extension reaction, salt removal using ion-exchange resin, and mass correlated genotype calling using SpectroCHIP array and MALDI-TOF mass spectrometry.

Serotonin transporter (5-HTTLPR) genotyping. 5-HTTLPR genotyping was performed at CHGR using Applied Biosystems' instruments and reagents. Genomic DNA was amplified using the following primer sequences: 5-HTTLPR-F 6FAM 5'GGCGTTGCCGCTCTGAATGC-3' and 5-HTTLPR-R 5'-GAGGGACTGAGCTGGACAACCAC- $3^{\prime}$. Amplified product was then combined with a size standard (GeneScan LIZ-500) before being analyzed. The 'long' allele appears as a product of approximately 528 base pairs, whereas the 'short' allele is approximately 483 base pairs.

Genetic ancestral cluster identification. We used a 40SNP Ancestry Informative Marker Set to derive genetic ancestral clusters using clustering tools provided in PLINK (Purcell et al, 2007). The Ancestry Informative Marker Set SNPs have very different minor allele frequencies across racial groups, and thus are informative for ancestry. They were designed to separate out the Caucasian, African, and Asian population blocks from each other, and they roughly map onto these self-reported racial groups in our sample (which are shown in Table 1). 
Table I Demographic Characteristics among Genetics Analysis Population

\begin{tabular}{|c|c|c|}
\hline \multirow[b]{2}{*}{ Site: N (\%) } & \multicolumn{2}{|c|}{$\begin{array}{l}\text { Patients with } \\
\text { genetic data } \\
(n=346)\end{array}$} \\
\hline & & \\
\hline $\mathrm{MGH}$ & 123 & $36 \%$ \\
\hline UCSD & 104 & $30 \%$ \\
\hline McMaster & 119 & $34 \%$ \\
\hline Age at entry: mean (SD), years & 35 & $(\mid 3.3)$ \\
\hline \multicolumn{3}{|l|}{ Gender: N (\%) } \\
\hline Male & 230 & $66 \%$ \\
\hline Female & 116 & $34 \%$ \\
\hline \multicolumn{3}{|l|}{ Race (self-report): N (\%) } \\
\hline White & 268 & $77 \%$ \\
\hline Asian & 27 & $8 \%$ \\
\hline Black/African American & 20 & $6 \%$ \\
\hline American Indian/Alaska Native/First Nations & 2 & $1 \%$ \\
\hline Other & 29 & $8 \%$ \\
\hline \multicolumn{3}{|l|}{ Ethnicity (self-report): N (\%) } \\
\hline Hispanic & 32 & $9 \%$ \\
\hline Non-hispanic & 314 & $91 \%$ \\
\hline \multicolumn{3}{|l|}{ Baseline LSAS: N (\%) } \\
\hline$<70$ & 44 & $13 \%$ \\
\hline$\geqslant 70$ & 302 & $87 \%$ \\
\hline \multicolumn{3}{|l|}{ Phase I instructions received: N (\%) } \\
\hline Exposure & 170 & $49 \%$ \\
\hline Neutral & 176 & $51 \%$ \\
\hline \multicolumn{3}{|l|}{ \# Comorbidities: N (\%) ${ }^{\mathrm{a}}$} \\
\hline 0 & 194 & $56 \%$ \\
\hline । & 98 & $28 \%$ \\
\hline 2 & 36 & $10 \%$ \\
\hline $3-4$ & 18 & $5 \%$ \\
\hline Lifetime alcohol abuse/dependence: N (\%) & 62 & $18 \%$ \\
\hline Age of sad onset: mean (SD) & 11 & $(6.8)$ \\
\hline MADRS total: mean (SD) & 11 & $(7.5)$ \\
\hline Avoidant PD \# criteria endorsed (7 total): mean (SD) & 4.4 & $(1.9)$ \\
\hline Sheehan disability total: mean (SD) & 16 & $(5.5)$ \\
\hline Week 10 sertraline blood levels (ng/ml): mean (SD) & 68 & $(46.7)$ \\
\hline Completed phase I: N (\%) & 273 & $79 \%$ \\
\hline
\end{tabular}

Includes MDD, Panic, OCD, and GAD

\section{Statistical Approach}

An additive genetic model was used for the primary analysis for each variant, corresponding to a single-predictor variable for the variant in the model, coded as 0 , 1 , or 2 to reflect the number of minor alleles in a subject's genotype (see Table 2 for tabulations of number of minor alleles for each gene variant). The main effect for each variant is that of adding one additional minor allele to the genotype (comparing 1 minor allele to 0 as well as 2 minor alleles to 1 ). The use of an additive model is common in genetic association studies because the true underlying model is seldom known and an additive model is considered to be robust to model misspecification. Although genetic ancestral cluster was not associated with LSAS $(p=0.14)$, we nevertheless controlled for potential confounding by race as the genetic allele frequencies for some of the variants differed significantly by self-reported race.

We examined genetic predictors of change in LSAS total score over Phase I (measured at weeks 0, 1, 2, 4, 6, 8, 10) using mixed models with random effects for slope and intercept. Change in LSAS over time was non-linear, and therefore was modeled quadratically with terms for week and week ${ }^{2}$. We first univariately tested the predictive value of each genetic variant by using a likelihood ratio test to compare a model with and without the (variant $\times$ week) and $\left(\right.$ variant $\times$ week $^{2}$ ) interaction terms. To better interpret the results of this non-linear model, we calculated the effect of an increase of one minor allele on LSAS at week 10. In these univariate analyses of change in LSAS, we examined the spectral decomposition of the genetic variants using SNPSpD (Nyholt, 2004) to determine the number of effective independent comparisons being made, in order to control the type I error rate accordingly. The decomposition yielded six independent comparisons, and thus an $\alpha=0.05 / 6=0.00833$ was applied, based on the Bonferroni correction. Finally, we added number of current comorbidities (the only significant clinical predictor of change in LSAS over time, categorized as $0,1,2,3,4)$ and genetic ancestral cluster into the univariate model for each significant genetic variant to determine if the significance was maintained.

\section{RESULTS}

Table 3 shows the results for each of the genetic variants tested. Based on the likelihood ratio test simultaneously testing the (variant $\times$ week) and (variant $\times$ week $^{2}$ ) interaction terms, we found that all four RGS2 SNPs showed studywise significance $(\alpha=0.00833)$ when tested univariately. These four RGS2 SNPs are all in high linkage disequilibrium with each other (all pairwise $R^{2}>0.8$ in the study sample), and thus were not entered together into a multivariate model. Two haplotypes of these four SNPs are common: (1) TGGC - the all minor alleles haplotype of rs10801152rs6428136-rs4606-rs1819741, with a frequency of $\sim 25 \%$ in the study sample, and (2) ATCT - the all major alleles haplotype, with a frequency of $\sim 72 \%$. These two haplotypes were also tested for association with change in LSAS over Phase I, and both were significantly associated with change in LSAS, with the TGGC haplotype even more so than the best individual RGS2 SNP, rs1819741. After controlling for number of current comorbidities and genetic ancestral cluster, significance of all four RGS2 SNPs as well as both haplotypes was maintained.

Figure 1 shows of the direction of effects of all four RGS2 SNPs (Figure 1a-d), as well as the TGGC (Figure 1e) and ATCT (Figure 1f) haplotypes. For the RGS2 SNPs and the TGGC haplotype, the minor allele was associated with smaller changes in LSAS total score over time, whereas for 
Table 2 Frequency of Number of Minor Alleles by Gene Variant

\begin{tabular}{|c|c|c|c|c|c|c|}
\hline SNP & Minor allele & Gene symbol & Gene name & 0 Copies (\%) & I Copy (\%) & 2 Copies (\%) \\
\hline rs 10801152 & $\mathrm{~T}$ & RGS2 & Regulator of $\mathrm{G}$ protein signaling 2 & 51 & 41 & 8 \\
\hline rs6428136 & G & RGS2 & Regulator of $\mathrm{G}$ protein signaling 2 & 55 & 38 & 7 \\
\hline rs4606 & G & RGS2 & Regulator of $\mathrm{G}$ protein signaling 2 & 52 & 39 & 9 \\
\hline rs|819741 & C & RGS2 & Regulator of $G$ protein signaling 2 & 54 & 38 & 8 \\
\hline rs3742278 & C & HTR2A & Serotonin $2 \mathrm{~A}$ receptor & 71 & 26 & 3 \\
\hline 5-HTTLPR & S & SLC6A4 & Serotonin transporter & 24 & 51 & 25 \\
\hline TGGC haplotype & - & - & All minor alleles haplotype & 57 & 36 & 7 \\
\hline ATCT haplotype & - & - & All major alleles haplotype & 8 & 41 & 50 \\
\hline
\end{tabular}

Table 3 Genetic Variants as Predictors of Phase I (10-week) Change in LSAS

\begin{tabular}{|c|c|c|c|c|c|}
\hline SNP $\mathbf{P}^{\mathrm{b}}$ & Minor allele & Gene symbol & Gene name & Week 10 estimate (SE) ${ }^{c}$ & LRT $p$-value ${ }^{d}$ \\
\hline rs $1080 \mid 152$ & $\mathrm{~T}$ & RGS2 & Regulator of $\mathrm{G}$ protein signaling 2 & $2.68(2.36)$ & $0.0045 *$ \\
\hline rs6428I36 & G & RGS2 & Regulator of $\mathrm{G}$ protein signaling 2 & $2.89(2.42)$ & $0.0043 *$ \\
\hline rs4606 & G & RGS2 & Regulator of $\mathrm{G}$ protein signaling 2 & $3.08(2.36)$ & 0.005 I* \\
\hline rs|8|974| & C & RGS2 & Regulator of $\mathrm{G}$ protein signaling 2 & $3.54(2.37)$ & $0.0025 *$ \\
\hline rs3742278 & C & HTR2A & Serotonin $2 \mathrm{~A}$ receptor & ।.31 (2.87) & 0.029 \\
\hline 5-HTTLPR & S & SLC6A4 & Serotonin transporter & $0.44(2.25)$ & 0.39 \\
\hline TGGC haplotype & - & - & All minor alleles haplotype & $3.56(2.42)$ & $0.0025 *$ \\
\hline ATCT haplotype & - & - & All major alleles haplotype & $-2.46(2.38)$ & $0.0068 *$ \\
\hline
\end{tabular}

* $p$-value $<0.05 / 6=0.00833$ after principal components analysis and Bonferroni correction.

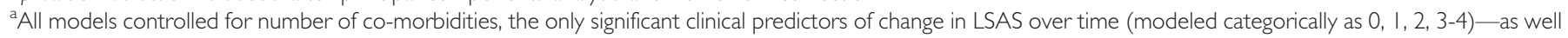
as genetic ancestral cluster (which roughly mapped onto White, Black, Asian, all other).

bEach variant was modeled linearly as 0, I, 2 minor alleles (ie, additive model was used).

${ }^{c}$ The estimate is based on the longitudinal model and represents the difference in week 10 LSAS total score when the number of minor alleles is increased by 1.

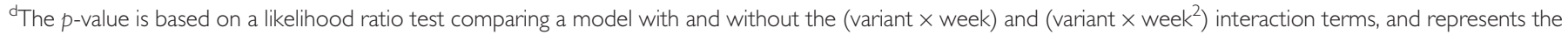
effect of each variant on the change in LSAS over time.

the ATCT haplotype, the minor allele was associated with larger gains over time.

Given the findings with change in LSAS, we decided to examine whether variants of the RGS2 gene and the TGGC and ATCT haplotypes predicted Phase I response and remission in the ITT population (ie response and remission were based on the last Phase I LSAS score for patients who terminated early) using logistic regression. Spectral decomposition of the genetic variants (to control the type I error rate; Nyholt, 2004) yielded two independent comparisons, and thus we used an $\alpha$ of 0.025 for these analyses, based on the Bonferroni correction. We also examined the nongenetic covariates shown in Table 1 as predictors of response using univariate logistic regression (at the $\alpha=0.05$ type I error rate), and subsequently controlled for any significant clinical predictors in our models examining genetic predictors of response and remission.

As seen in Table 4, none of the genetic variants predicted Phase I response after controlling for genetic ancestral cluster and significant clinical predictors of response, which included (1) Avoidant PD Score (number of questions, out of 7 , scored as a $3=$ threshold/true); (2) lifetime alcohol abuse or dependence (Yes/No); and (3) impairment severity (sum of Sheehan Disability (Leon et al, 1997) scores for work, social life, and family life/home responsibilities). However, the SNP rs4606 was a significant predictor of Phase I remission $(p<0.025)$, rs1819741 was borderline significant $(p=0.027)$, and the TGGC haplotype was nominally significant $(p<0.05)$.

\section{DISCUSSION}

SSRIs are the most widely prescribed evidence-based form of pharmacotherapy for SAD (Stein and Stein, 2008). Despite their widespread use, only approximately $50 \%$ of adult patients with SAD derive significant therapeutic benefit. In the present controlled study-the largest ever to provide standardized SSRI treatment to patients with SAD - the response rate was low (33\%) and the remission 
a

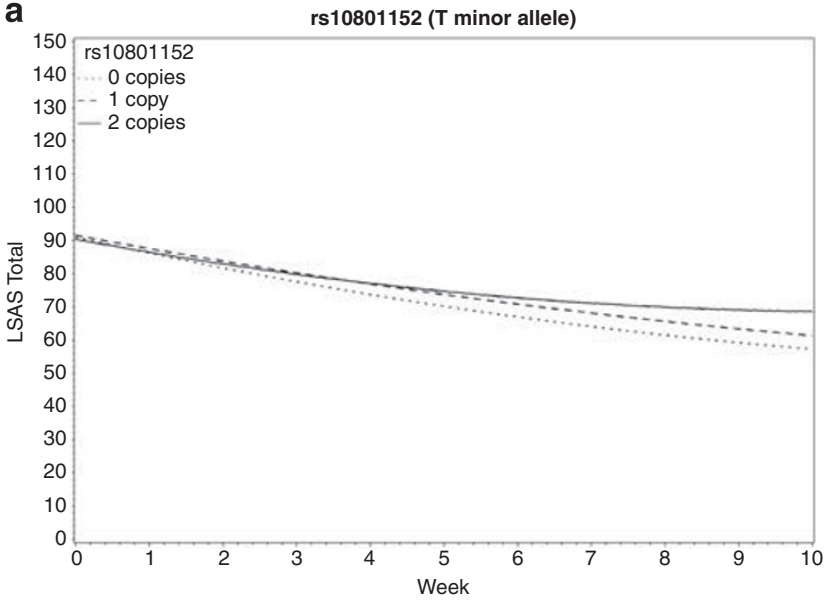

C
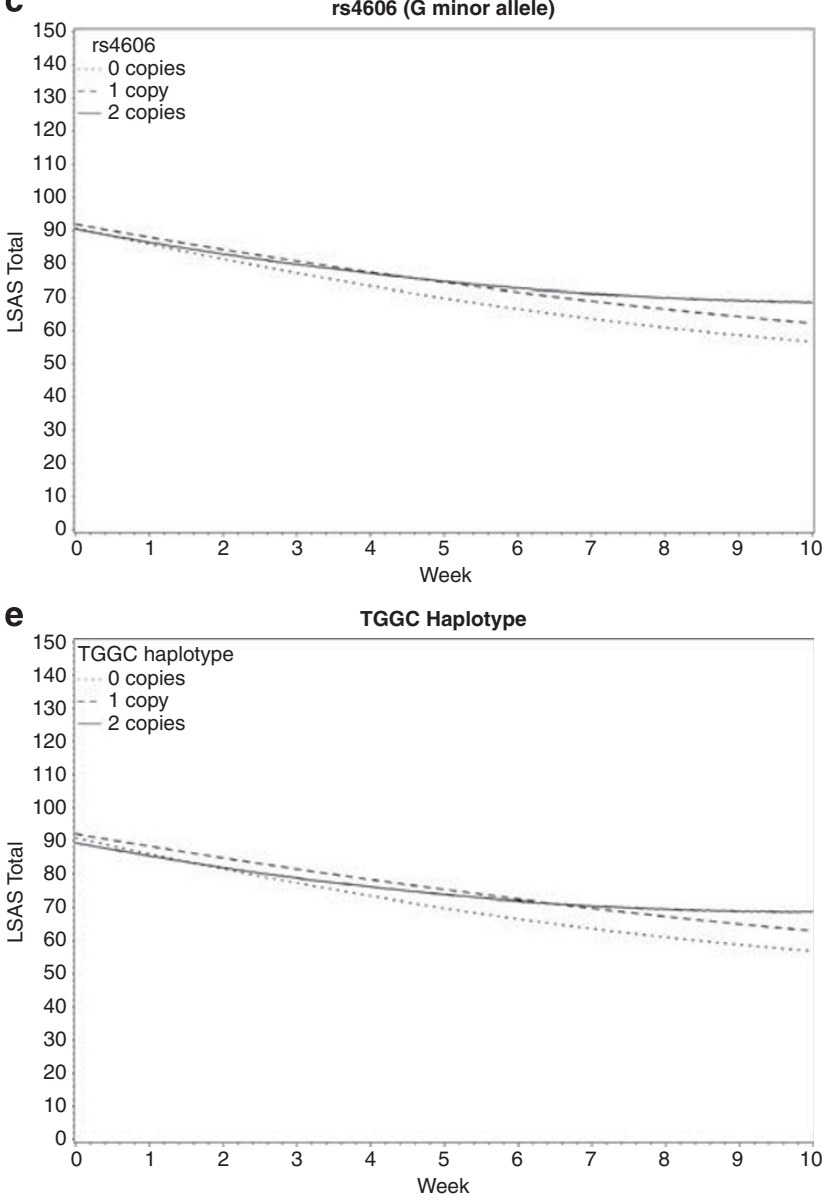

b

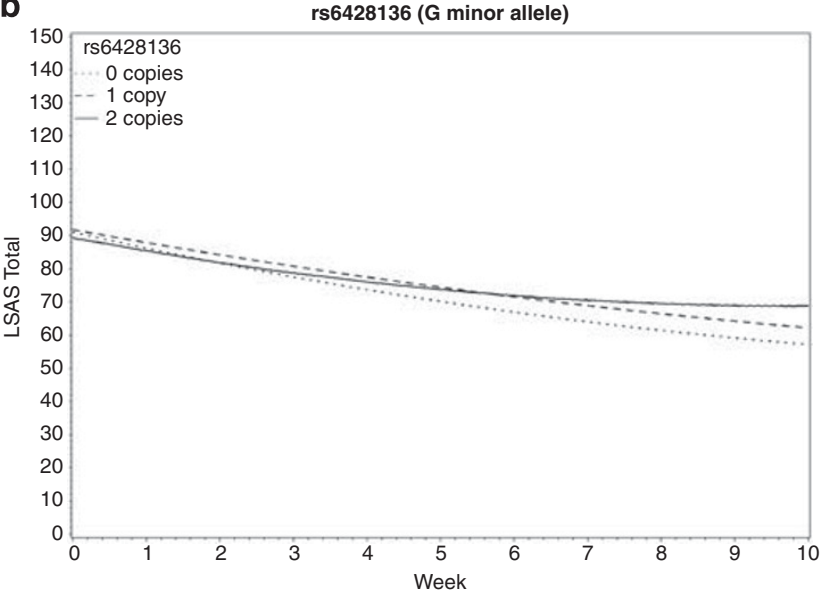

d

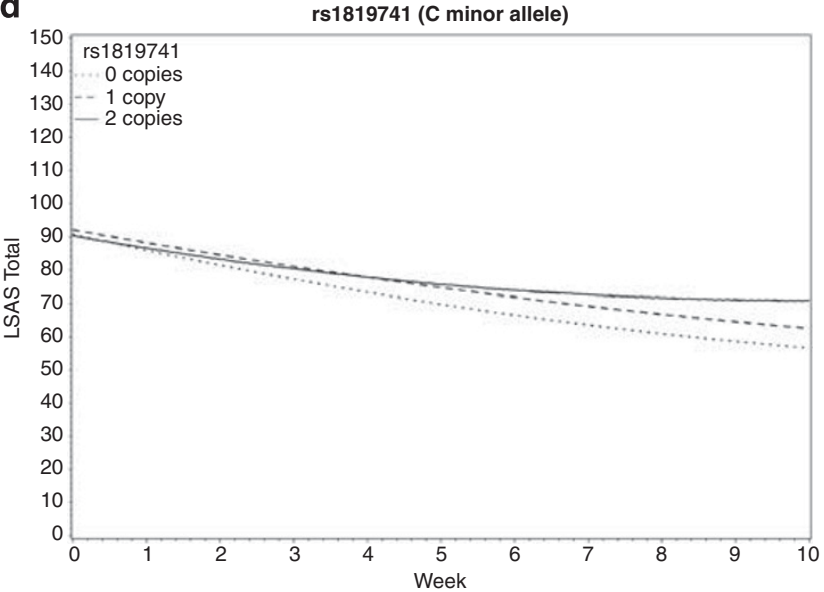

f

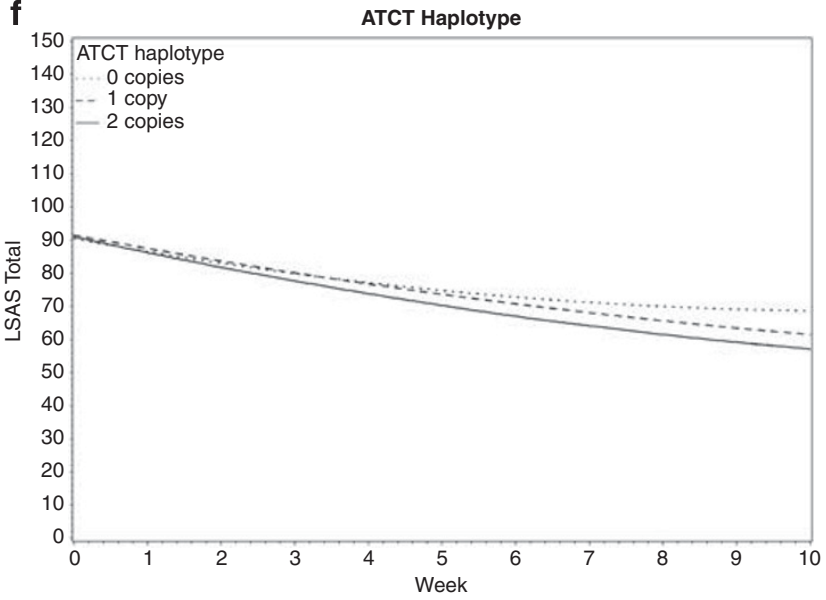

Figure I Change in Liebowitz Social Anxiety Disorder (LSAS) total score over Phase I by minor allele copies for select SNPs. Shown are predicted trajectories of symptom (LSAS) change over time in patients with social anxiety disorder receiving selective serotonin reuptake inhibitor treatment, stratified by number of copies of minor allele in each single-nucleotide polymorphism as noted. Predicted values were based on a quadratic model (ie, LSAS $\sim \beta_{0}+\beta_{1}$ week $+\beta_{2}$ week $^{2}$.

rate even lower (13\%). These data speak to the need for predictive biomarkers of SSRI response in SAD with the aim of identifying a priori who is more or less likely to respond before embarking on a therapeutic trial.

In the present study, looking at a very limited, prespecified panel of potential genetic predictors, we found that several markers in RGS2 predicted less reduction in symptoms and lower likelihood of remission with SSRI treatment. We chose to look at RGS2 variants because of their association with the phenotype SAD-related traits such as introversion and behavioral inhibition, as well as functional MRI markers such as enhanced amygdala and 
Table 4 Select Genetic Variants as Predictors of Phase I Response and Remission

\begin{tabular}{|c|c|c|c|c|c|c|c|}
\hline \multirow[t]{2}{*}{ SNPa } & \multirow[t]{2}{*}{ Minor allele } & \multicolumn{3}{|c|}{ Phase I response $^{\mathrm{b}}$} & \multicolumn{3}{|c|}{ Phase I Remission ${ }^{\text {b }}$} \\
\hline & & Odds ratio & $(95 \% \mathrm{Cl})$ & $P$-value & Odds ratio & $(95 \% \mathrm{Cl})$ & $p$-value \\
\hline rs6428I36 & G & 0.79 & $(0.54-1.17)$ & 0.24 & 0.62 & $(0.36-1.12)$ & 0.10 \\
\hline rs4606 & G & 0.84 & $(0.58-1.23)$ & 0.37 & 0.49 & $(0.27-0.90)$ & $0.022 *$ \\
\hline ATCT haplotype & - & 1.13 & $(0.78-1.65)$ & 0.52 & 1.73 & $(0.99-3.05)$ & 0.063 \\
\hline
\end{tabular}

*p-value $<0.05 / 2=0.025$ after principal components analysis and Bonferroni correction

aEach variant was modeled linearly as 0, 1, 2 minor alleles (ie, additive model was used).

${ }^{b}$ All models controlled for genetic ancestral cluster (which roughly mapped onto White, Black, Asian, all other) as well as significant clinical predictors of response, ie, Avoidant PD Score (number of questions, out of 7, scored as a 3 =threshold/true); lifetime alcohol abuse or dependence (yes/no); and impairment severity (sum of Sheehan Disability scores for work, social life, and family life/home responsibilities).

insula response to emotion faces (Smoller et al, 2008). The current findings are intriguing in light of the biological actions of the RGS2 protein. Among other functions, RGS2 accelerates the deactivation of $G$ proteins to reduce neuronal $G$ protein-coupled receptor response to neurotransmitters including serotonin (Kimple et al, 2011). Deletion of $r g s 2$ in mice produces increased anxiety-related behavior (Lifschytz et al, 2012; Oliveira-Dos-Santos et al, 2000; Yalcin et al, 2004) and avoidance behavior in novel social situations (Lifschytz et al, 2012). Mice lacking rgs2 have also been reported to show reduced expression of $5 \mathrm{HT}_{1 \mathrm{a}} \mathrm{G}$ protein-coupled presynaptic autoreceptors (thought to be involved in the therapeutic activity of SSRIs) in the raphe (Lifschytz et al, 2012). Of note, the G allele of rs4606, which we found to predict reduced sertraline response, has been associated with reduced expression of RGS2 (Semplicini et al, 2006). Along with our previous observation that this allele (and those in LD with it) are associated with temperamental indices of social anxiety (Smoller et al, 2008), these findings suggest that individuals carrying hypofunctional alleles of RGS2 may be relatively resistant to sertraline due to dysregulation of serotonergic neurotransmission and increased anxiety proneness. Of note, however, mutations of the G-protein subunit Gai2 that result in RGS insensitivity have been reported to enhance frontal cortex and hippocampal postsynaptic 5HT1a signaling and potentiate the antidepressant effect of the SSRI fluvoxamine in mice (Talbot et al, 2010). Thus, the effects of RGS2 on SSRI response may vary depending on anatomic and synaptic location. Further research is needed to clarify the mechanism through which RGS2 variants mediate SSRI response.

Strengths of the study are the fairly large sample size and the standardized protocol-driven treatment with a single SSRI (sertraline) and the systematic outcomes assessment. A limitation is the narrow focus on only a few candidate SNPs as opposed to a much broader (or even genomewide) approach such as has been applied to pharmacogenetic studies of major depressive disorder (Laje et al, 2009; Perlis, 2010). Given our findings with even this small set of markers, further attempts at the identification of genetic predictors of treatment response in SAD should be undertaken. In addition, although we observed statistically significant association of treatment outcomes with RGS2 genotypes, the predictive utility of these markers in clinical practice remains to be established. Another feature of the study that limits its clinical interpretation is the prediction of response to only one type of treatment, an SSRI. Whereas it is useful knowing that likelihood of remission to sertraline, as in the present study, is half as likely in some patients than others, it would be even more useful to know what those individuals would respond to. That, too, is a topic for future research. We consider the current findings to be an important step along the way to the identification of genomic predictors of treatment response in SAD, but are fully cognizant of the many steps that need to follow in the delineation of such biomarkers (Perlis, 2011), not the least of which is replication.

With regard to replicating, the present study failed to replicate the only other published finding of genetic prediction of SSRI response in SAD, where the short variant of 5HTTLPR had been associated with poorer response (Stein et al, 2006). Similar findings have occasionally, although by no means widely, seen in studies of SSRI response in major depression (Huezo-Diaz et al, 2009). We are unable to explain why the previous finding in SAD would not have been replicated in the present study, particularly given that the original finding was seen in a much smaller sample size. One possibility is that the prior study looked at response to a variety of SSRIs, whereas the present study looked solely at response to sertraline. Regardless, these observations speak to the need for replication before any conclusions about clinical utility can be drawn.

In summary, we found evidence that variants in RGS2 moderate the response to SSRIs in patients with SAD. Additional research is needed to replicate this finding, to place it in context of other yet-to-be-identified predictors of treatment response, and to determine the mechanism(s) by which these effects are seen. This line of research has the potential to lead to better personalization of treatments for patients with SAD and other mental disorders. 


\section{FUNDING AND DISCLOSURE}

Grant support was provided by the National Institute of Mental Health (NIMH), grant numbers MH070501, MH070919, and MH094614. Support for study drug and packaging was received from Pfizer and Wyeth Pharmaceuticals. Dr Stein reports the following disclosures: Grant support from NIMH, Department of Defense, Department of Veterans Affairs. He is co-holder of a patent on the use of genetic testing to predict treatment outcomes in social anxiety disorder. He receives payment for his work as CoEditor in Chief of UpToDate in Psychiatry, and for his work as Deputy Editor of Depression and Anxiety (Wiley Press). Dr Van Ameringen reports the following disclosures: Grant/ Research Support from Janssen-Ortho, National Institutes of Health (NIH), Pfizer; Speakers' Bureau for Valiant, GlaxoSmithKline, Lundbeck, Pfizer; he has also participated in Advisory Boards for Valiant, Eli Lilly, Janssen-Ortho, Labo Pharm, Lundbeck, Pfizer, and Shire. Dr Naomi Simon reports the following disclosures: NIH, Department of Defense, American Foundation for Suicide Prevention, Forest Research, MGH Psychiarty Academy, Highland Street Foundation, American Cancer Society, Glaxo SmithKline, Sepracor, Lilly, Pfizer, and NARSAD. Dr Pollack reports the following disclosures: Advisory Boards and Consultation: Brain Cells, Eli Lilly, Johnson and Johnson, Labopharm, Medavante, Mindsite, Otsuka, Pfizer, Sepracor, Targia, Transcept; Grant Support: Bristol Myers Squibb, Euthymics, Forest Laboratories, GlaxoSmithKline, Eli Lilly, NCCAM, NIDA, NIMH, Sepracor; CME activities: Astra-Zeneca, Sepracor, Pfizer; Royalty/Patents: SIGH-A, SAFER interviews; Equity: Doyen Medical, Medavante, Mensante Corporation, Mindsite, Targia Pharmaceuticals. Dr Smoller reports the following disclosure: Scientific Advisory Board membership of Psybrain. Stephen Haddad and Aparna Keshaviah report no competing interests.

\section{REFERENCES}

Arbelle S, Benjamin J, Golin M, Kremer I, Belmaker RH, Ebstein RP (2003). Relation of shyness in grade school children to the genotype for the long form of the serotonin transporter promoter region polymorphism. Am J Psychiatry 160: 671-676.

Fresco DM, Coles ME, Heimberg RG, Liebowitz MR, Hami S, Stein $\mathrm{MB}$ et al (2001). The Liebowitz Social Anxiety Scale: a comparison of the psychometric properties of self-report and clinician-administered formats. Psychol Med 31: 1025-1035.

Gelernter J, Page GP, Stein MB, Woods SW (2004). Genome-wide linkage scan for loci predisposing to social phobia: evidence for a chromosome 16 risk locus. Am J Psychiatry 161: 59-66.

Huezo-Diaz P, Uher R, Smith R, Rietschel M, Henigsberg N, Marusic A et al (2009). Moderation of antidepressant response by the serotonin transporter gene. Br J Psychiatry 195: 30-38.

Kimple AJ, Bosch DE, Giguere PM, Siderovski DP (2011). Regulators of G-protein signaling and their Galpha substrates: promises and challenges in their use as drug discovery targets. Pharmacol Rev 63: 728-749.

Laje G, Perlis RH, Rush AJ, McMahon FJ (2009). Pharmacogenetics studies in $\mathrm{STAR}^{\star} \mathrm{D}$ : strengths, limitations, and results. Psychiatr Serv 60: 1446-1457.

Leon AC, Olfson M, Portera L, Farber L, Sheehan DV (1997). Assessing psychiatric impairment in primary care with the Sheehan Disability Scale. Int J Psychiatry Med 27: 93-105.
Lifschytz T, Broner EC, Zozulinsky P, Slonimsky A, Eitan R, Greenbaum L et al (2012). Relationship between Rgs2 gene expression level and anxiety and depression-like behaviour in a mutant mouse model: serotonergic involvement. Int J Neuropsychopharmacol 15: 1307-1318.

Masi G, Pfanner C, Mucci M, Berloffa S, Magazu A, Parolin G et al (2012). Pediatric social anxiety disorder: predictors of response to pharmacological treatment. J Child Adolesc Psychopharmacol 22: $410-414$.

Nyholt DR (2004). A simple correction for multiple testing for single-nucleotide polymorphisms in linkage disequilibrium with each other. Am J Hum Genet 74: 765-769.

Oliveira-Dos-Santos AJ, Matsumoto G, Snow BE, Bai D, Houston FP, Whishaw IQ et al (2000). Regulation of T cell activation, anxiety, and male aggression by RGS2. Proc Natl Acad Sci USA 97: 12272-12277.

Perlis RH (2010). Can single genes matter in a polygenic world? Biol Psychiatry 68: 783-784.

Perlis RH (2011). Translating biomarkers to clinical practice. Mol Psychiatry 16: 1076-1087.

Pollack MH, Van Ameringen M, Simon NM, Worthington JW, Hoge EA, Keshaviah A et al (2013). A double-blind randomized controlled trial of augmentation and switch strategies for refractory social anxiety disorder. Am J Psychiatry (in press).

Purcell S, Neale B, Todd-Brown K, Thomas L, Ferreira MA, Bender $\mathrm{D}$ et al (2007). PLINK: a tool set for whole-genome association and population-based linkage analyses. Am J Hum Genet 81: 559-575.

Ruscio AM, Brown TA, Chiu WT, Sareen J, Stein MB, Kessler RC (2008). Social fears and social phobia in the USA: results from the National Comorbidity Survey Replication. Psychol Med 38: $15-28$.

Semplicini A, Lenzini L, Sartori M, Papparella I, Calo LA, Pagnin E et al (2006). Reduced expression of regulator of G-protein signaling 2 (RGS2) in hypertensive patients increases calcium mobilization and ERK1/2 phosphorylation induced by angiotensin II. J Hypertens 24: 1115-1124.

Smoller JW, Paulus MP, Fagerness JA, Purcell S, Yamaki LH, Hirshfeld-Becker D et al (2008). Influence of RGS2 on anxietyrelated temperament, personality, and brain function. Arch Gen Psychiatry 65: 298-308.

Stein DJ, Ipser JC, Balkom AJ (2004). Pharmacotherapy for social phobia. Cochrane Database Syst Rev CD001206.

Stein MB, Seedat S, Gelernter J (2006). Serotonin transporter gene promoter polymorphism predicts SSRI response in generalized social anxiety disorder. Psychopharmacology (Berl) 187: 68-72.

Stein MB, Stein DJ (2008). Social anxiety disorder. Lancet 371: 1115-1125.

Talbot JN, Jutkiewicz EM, Graves SM, Clemans CF, Nicol MR, Mortensen RM et al (2010). RGS inhibition at G(alpha)i2 selectively potentiates 5-HT1A-mediated antidepressant effects. Proc Natl Acad Sci USA 107: 11086-11091.

Uher R, Tansey KE, Malki K, Perlis RH (2012). Biomarkers predicting treatment outcome in depression: what is clinically significant? Pharmacogenomics 13: 233-240.

Van Ameringen M, Oakman J, Mancini C, Pipe B, Chung H (2004). Predictors of response in generalized social phobia: effect of age of onset. J Clin Psychopharmacol 24: 42-48.

Wagner KD, Berard R, Stein MB, Wetherhold E, Carpenter DJ, Perera $\mathrm{P}$ et al (2004). A multicenter, randomized, double-blind, placebo-controlled trial of paroxetine in children and adolescents with social anxiety disorder. Arch Gen Psychiatry 61: 1153-1162.

Yalcin B, Willis-Owen SA, Fullerton J, Meesaq A, Deacon RM, Rawlins JN et al (2004). Genetic dissection of a behavioral quantitative trait locus shows that Rgs2 modulates anxiety in mice. Nat Genet 36: 1197-1202. 